\title{
LDL electronegativity index: a potential novel index for predicting cardiovascular disease
}

This article was published in the following Dove Press journal:

Vascular Health and Risk Management

28 August 2015

Number of times this article has been viewed

Ekaterina A Ivanova'

Yuri V Bobrysheve,

Alexander N Orekhov ${ }^{2,4,5}$

'Department of Pediatric Nephrology and Growth and Regeneration, Katholieke Universiteit Leuven and University Hospitals Leuven, Leuven, Belgium; 'Laboratory of Angiopathology, Institute of General Pathology and Pathophysiology, Russian Academy of Sciences, Moscow, Russia; ${ }^{3}$ Faculty of Medicine, School of Medical Sciences, University of New South Wales, Kensington, Sydney, NSW, Australia; ${ }^{4}$ Institute for Atherosclerosis Research, Skolkovo Innovative Center, Moscow, Russia; ${ }^{5}$ Department of Biophysics, Faculty of Biology, Lomonosov Moscow State University, Moscow, Russia
Correspondence: Yuri V Bobryshev Faculty of Medicine, University of New South Wales (UNSW), High Street, Sydney, NSW 2052, Australia

Tel $+6 \mid 293851217$

Fax +6I 293851217

Email y.bobryshev@unsw.edu.au
Abstract: High cardiovascular risk conditions are frequently associated with altered plasma lipoprotein profile, such as elevated low-density lipoprotein (LDL) and LDL cholesterol and decreased high-density lipoprotein. There is, however, accumulating evidence that specific subclasses of LDL may play an important role in cardiovascular disease development, and their relative concentration can be regarded as a more relevant risk factor. LDL particles undergo multiple modifications in plasma that can lead to the increase of their negative charge. The resulting electronegative LDL $[\operatorname{LDL}(-)]$ subfraction has been demonstrated to be especially atherogenic, and became a subject of numerous recent studies. In this review, we discuss the physicochemical properties of LDL(-), methods of its detection, atherogenic activity, and relevance of the LDL electronegativity index as a potential independent predictor of cardiovascular risk.

Keywords: low-density lipoprotein, LDL, LDL electronegativity index, cardiovascular disease, atherosclerosis

\section{Introduction}

The risk of cardiovascular disease (CVD) development is closely associated with altered lipoprotein profile of blood plasma. Lipoprotein particles circulating in human blood vary in their chemical and physical properties. It is well documented that individuals with high CVD risk have elevated amounts of circulating low-density lipoprotein (LDL) and decreased proportion of high-density lipoprotein (HDL). LDL is the major source of cholesterol and the lipids that contribute to the development of the atherosclerotic plaque, whereas HDL is not atherogenic, and its concentrations inversely correlate with the CVD risk. ${ }^{1}$ Total cholesterol and LDL cholesterol lowering with statins is therefore standard therapeutic approach for treating patients with atherosclerosis and increased CVD risk. ${ }^{2,3}$ This strategy, however, did not reduce the CVD risk beyond $\sim 70 \%$ in most cases, and in some clinical studies lowering LDL cholesterol was not efficient..$^{47}$ This pointed to the existence of other important factors promoting atherosclerosis development that have to be taken into account.

The LDL pool is heterogenic and consists of several subclasses that can be separated based on the differences in their density, size, chemical composition, and electrical charge. ${ }^{8,9}$ Among them, small dense LDL (sdLDL) was demonstrated to be highly atherogenic, and its level strongly correlated with CVD risk. ${ }^{10-12}$ Study of the significance of LDL subclasses for atherosclerosis progression has been hindered by the lack of standardization of the analytical methods. LDL subfraction analysis has been performed by different groups, using gradient density ultracentrifugation, ${ }^{13-15}$ 
gradient gel electrophoresis, ${ }^{16}$ nuclear magnetic resonance (NMR) analysis, ${ }^{17}$ homogeneous assays, ${ }^{18-20}$ and other methods. All these methods deliver slightly different results, and variations are possible even within one method due to the modifications of experimental conditions..$^{21,22}$ It is, however, generally accepted that sdLDL fraction has a density of $1.044-1.063 \mathrm{~g} / \mathrm{mL}$ and a particle size around $15-20 \mathrm{~nm}^{23}$ LDL subclasses analysis is currently being considered as an important diagnostic tool for improvement of CVD risk and treatment efficacy assessment. ${ }^{24}$

Apart from the size and density, LDL subfractions differ by their chemical composition. LDL particles can undergo multiple modifications in the blood plasma that increase their atherogenicity. ${ }^{25-27}$ One of the first types of atherogenic modified LDL that has been discovered is oxidation. ${ }^{25,28}$ Oxidized LDL particles are recognized by a number of receptors, including CD36 and TLR-4 and can induce the immune response and inflammation that contribute to the atherosclerosis progression. ${ }^{29,30}$ Oxidized LDL was demonstrated to induce the lipid storage in cultured endothelial cells (ECs), but could only be prepared in vitro. ${ }^{31,32}$

Other forms of modified LDL have been discovered during the last two decades. Desialylated LDL could be detected in the blood plasma of atherosclerosis patients using a lectinsorbent assay. ${ }^{33,34}$ Desialylation of LDL particles is performed in plasma by trans-sialidase that participates in the metabolism of glycoconjugates. ${ }^{35}$ Glycation of apoB lipoprotein in LDL particles has also been described. ${ }^{36,37}$

Another form of atherogenic modified LDL is electronegative LDL $[\operatorname{LDL}(-)]$ that can be distinguished using methods sensitive to the particle charge, such as agarose gel electrophoresis, isotachophoresis, or ion-exchange chromatography. ${ }^{38,39} \mathrm{LDL}(-)$ is characterized by an enhanced ability to aggregate and is associated with the increased CVD risk. ${ }^{40}$

It has been demonstrated that sdLDL is especially susceptible to atherogenic modifications that occur in blood plasma. sdLDL particles have a much longer circulation time than larger LDL subfractions that are cleared from the bloodstream through interaction with the LDL receptor. ${ }^{11,41}$ For instance, glycation of LDL particles was observed preferentially in the sdLDL fraction, and small LDL had a decreased sialic acid content. ${ }^{42,43}$ sdLDL particles contained less antioxidative vitamins that make them less protected against oxidation. ${ }^{44}$ Moreover, the elevated LDL(-) was associated with higher oxidized LDL and sdLDL levels..$^{45}$ Isolated LDL(-) particles had a decreased sialic acid content in comparison with native LDL, and this difference was more pronounced in $\operatorname{LDL}(-)$ fractions from patients with atherosclerosis. ${ }^{27,46}$ It is likely that desialylated and electronegative LDL fractions are closely linked or even identical. Converging evidence points to the existence of a cascade of multiple modifications of sdLDL particles, starting with desialylation and minimal oxidation, followed by further oxidation and formation of highly atherogenic and proinflammatory complexes.

\section{LDL electronegativity index}

LDL(-) is a heterogeneous population of particles that vary by their density and size, but share the increased negative charge..$^{47}$ The origins of LDL(-) are not yet clearly understood. Incubation of LDL with blood plasma at $37^{\circ} \mathrm{C}$ resulted in the formation of $\operatorname{LDL}(-)$ that could be blocked by 4-bromophenacyl bromide, an inhibitor of secretory phospholipase $\mathrm{A}_{2}\left(\mathrm{sPLA}_{2}\right)$, suggesting that $\mathrm{SPLA}_{2}$ plays a primary role in LDL(-) formation. ${ }^{48}$ Desialylation of LDL during incubation with blood plasma has also been described. ${ }^{35}$ It is likely that the acquisition of the negative charge occurs as a result of successive changes in the LDL particle, including desialylation, loss of lipids, reduction of particle size, and peroxidation in the blood plasma. A summary of such modifications leading to the formation of $\operatorname{LDL}(-)$ is presented in Figure 1.

\section{LDL(-) detection}

The separation of LDL into electropositive $[\operatorname{LDL}(+)]$ and electronegative [LDL(-)] fractions was first performed by Avogaro et $\mathrm{al}^{39}$ using ion-exchange chromatography. More recently, Chen et $\mathrm{al}^{49}$ described five subfractions of plasma LDL with various degrees of electronegativity, from L1 (least electronegative) to L5 (most electronegative). ${ }^{50}$ The authors found that the plasma levels of the most electronegative (L5) fraction were moderately elevated in individuals with high CVD risk, such as smokers, patients with hypercholesterolemia, type 2 diabetes, and myocardial infarction. ${ }^{51-53}$ Anion exchange chromatography remains the standard method for LDL(-) extraction for further analysis that allows a more detailed study of chemical and physical properties of these particles. Another approach to analyze the LDL subfractions based on the particle charge is the capillary isotachophoresis. ${ }^{54}$ In this method, LDL(-) is detected as a fraction of fast migrating LDL, which is separated from slowly migrating LDL. Some authors used capillary isotachophoresis to analyze the sdLDL fraction obtained by heparin precipitation. ${ }^{55}$ Finally, recently created monoclonal antibodies to LDL(-) allows distinguishing the LDL(-) fraction from native LDL particles by the specific epitopes. ${ }^{9,56,57}$ 


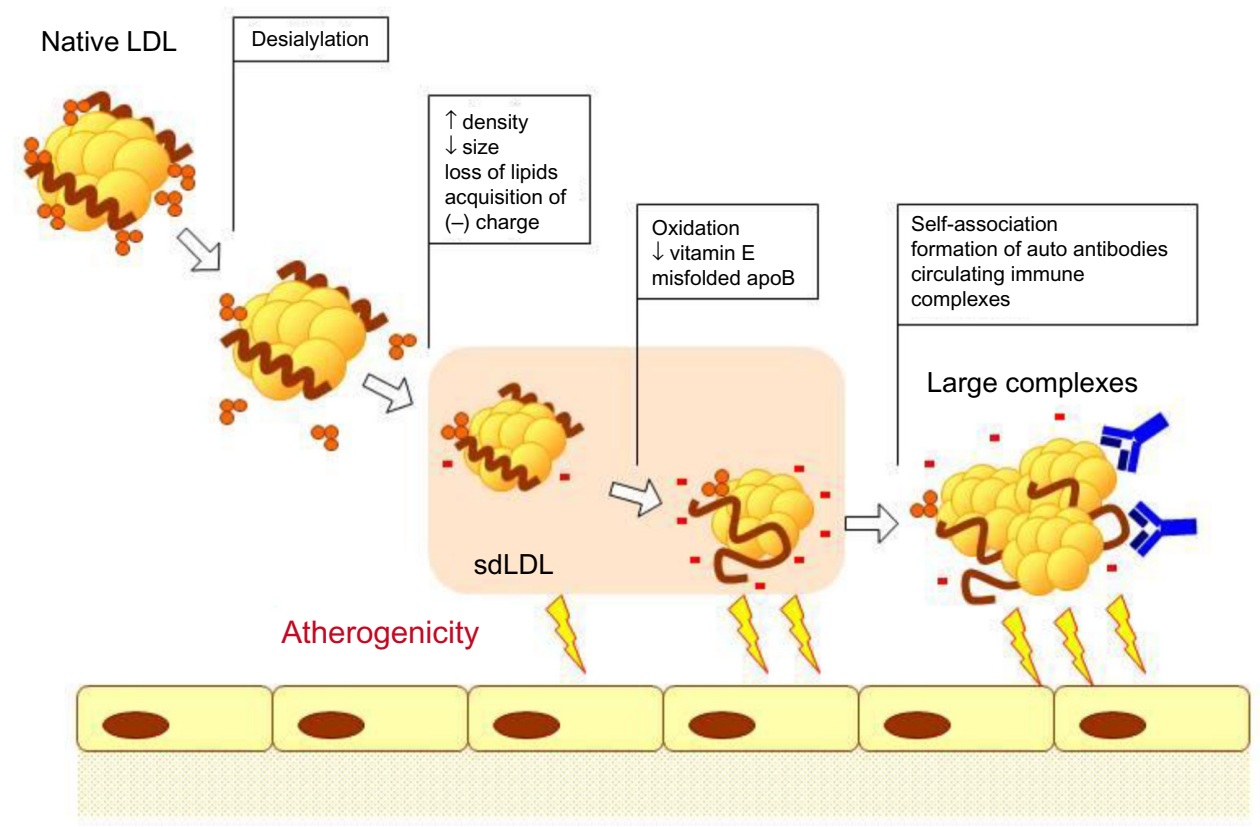

Figure I Atherogenic modifications of LDL.

Notes: Multiple atherogenic modifications of LDL particles have been detected in human blood plasma: desialylation was the first event, followed by loss of free cholesterol and cholesterol esters, phospholipids and triglycerides, increase of particle density, and decrease of its size; next, negative charge of particles was increased, leading to the formation of electronegative LDL fraction, in which misfolded apoB was reported; at later stages, increased oxidation and decreased antioxidant content were observed; finally, large, highly atherogenic complexes were formed due to self-association of modified LDL particles and the formation of autoantibodies.

Abbreviations: LDL, low-density lipoprotein; sdLDL, small dense LDL.

Enzyme-linked immunosorbent assay (ELISA) assay with monoclonal antibodies is especially useful for fast and direct detection of $\operatorname{LDL}(-)$ in clinical practice. ${ }^{58}$ It has to be taken into account that the existing $\operatorname{LDL}(-)$ detection methods are only sensitive to the electrical charge and do not distinguish the different origins of the LDL(-) particles. For instance, they cannot discriminate particles that vary by the relative amount of lipoproteins and/or nonesterified fatty acids, particles that underwent oxidation etc. Most of the available monoclonal antibodies were generated against LDL(-) particles isolated based on their charge by ion-exchange chromatography, and they do not help to overcome this problem.

\section{Tendency to aggregate}

LDL(-) particles are characterized by a number of specific chemical and physical features in comparison to native LDL. It is well known that $\operatorname{LDL}(-)$ is prone to aggregation. ${ }^{39}$ Spontaneous aggregation of LDL(-) particles has been investigated in more detail in a recent study. ${ }^{59}$ The authors report that LDL(-) formed amyloid-like structures and even possessed amyloidogenic properties, promoting native LDL particles to aggregate. Moreover, amyloid- $\beta$ peptide enhanced the aggregation of LDL(-) particles. LDL particle aggregation is closely related to their atherogenic activity, and aggregated LDL(-) is characterized by an increased proteoglycan binding. ${ }^{60,61}$
The nature of this increased affinity is currently being investigated. Some authors proposed that the N-terminus of apoB in the LDL(-) particles play the key role as monoclonal antibodies to this region interfered with the binding of particles to proteoglycans. ${ }^{62}$ Thus, this region could be an additional proteoglycan-binding site present in $\operatorname{LDL}(-)$, next to the constitutive binding site in apoB from native LDL. ${ }^{63,64}$

\section{Misfolded apoB}

The electronegative L5 fraction had a decreased content of apolipoprotein B (apoB) and increased content of other lipoproteins. ${ }^{65}$ A misfolded conformation of apoB in LDL(-) particles has also been reported. ${ }^{66,67}$ As demonstrated by circular dichroism studies, secondary structure of apoB is disturbed in LDL(-) particles, with decreased content of $\alpha$-helices and increased content of $\beta$-sheets. ${ }^{68}$ Studies of tryptophan fluorescence spectroscopy also pointed to the misfolded conformation of apoB, as the fluorescence emission was decreased in LDL(-) in comparison to native LDL, indicative of the abnormal exposure of tryptophan residues to the aqueous environment. ${ }^{59,68}$ Another evidence of lipoprotein misfolding in LDL(-) particles comes from 2D-NMR analysis that demonstrated a number of lysine residues in LDL(-) with altered ionization status probably due to their exposure to the solvent. ${ }^{69}$ Interestingly, estradiol prevented the misfolding of 
apoB in LDL(-) particles and decreased their aggregation ability without affecting the negative charge. ${ }^{70}$ Therefore, the misfolded lipoprotein appears to be the decisive factor responsible for $\operatorname{LDL}(-)$ aggregation. $\operatorname{LDL}(-)$ fractions also demonstrate changes in their lipid composition, such as increased content of nonesterified fatty acid. ${ }^{71,72}$ This may explain the altered properties of LDL(-) particles' surface and contribute to their enhanced aggregation ability. ${ }^{73}$

\section{Lipolytic activity}

LDL(-) particles are characterized by the enzymatic activity that modulates their lipid composition and inflammatory properties. It has been demonstrated that the particles are enriched with platelet-activating factor acetylhydrolase (PAF-AH), a phospholipase that targets the oxidized phospholipids. ${ }^{74}$ PLC-like and SMase activities have also been detected in LDL particles, although their origins remain to be elucidated. ${ }^{75,76}$ Proteomic studies detected a higher content of non-apoB proteins in $\operatorname{LDL}(-)$ in comparison to native LDL, including apoA-I, apoE, apoC-III, apoA-II, apoD, apoF, and apoJ. ${ }^{77}$ Although the absolute amounts of these proteins remain very low, they could still play a role in LDL(-) properties and lipolytic activities. For instance, ApoJ acts as a chaperone that binds the misfolded proteins in blood. ${ }^{78}$ Its binding to the misfolded apoB in LDL(-) particles could have a protective role against particle aggregation. The combination of PAF-AH and PLC-like activities could regulate the concentrations of proinflammatory oxidized LDL to attenuate their deleterious effects in human plasma. ${ }^{64}$ More studies are needed however to determine the origin and significance of these lipolytic activities.

\section{Inflammatory and atherogenic properties}

Several features of LDL(-) account for the increased atherogenicity of this LDL subtype (Figure 2). Altered protein structure of the LDL(-) particles leads to a decreased affinity to the LDL receptor as compared to native LDL and prolonged circulation times of the particles. ${ }^{79,80}$ On the other hand, the most electronegative (L5) fraction of LDL(-) was shown to interact with the lectin-like oxidized LDL receptor 1 (LOX-1), causing endothelial dysfunction, apoptosis, and atherogenic response in cultured ECs. ${ }^{51,81}$ A recent study demonstrated that L5 LDL(-) fraction induced the production of reactive oxygen species and increased the $\mathrm{C}$-reactive protein levels in cultured cells via LOX-1 signaling in cultured aortic ECs, which can further contribute to the atherogenesis. ${ }^{82}$ Circulating LDL(-) can activate inflammatory and immune responses that contribute to the atherosclerosis progression. Because of its increased ability to bind proteoglycans, LDL(-) particles and their aggregates have a prolonged residence time in the subendothelial space. Internalized by macrophages through scavenger receptors, LDL(-) serves as a source for the lipid storage and foam cell formation in the arterial wall. The production of anti-LDL(-) autoantibodies may also play an important role in the disease progression, which currently remains to be studied. ${ }^{9}$ Along with the autoantibodies to LDL, LDL-containing circulating immune complexes have been found in blood plasma of atherosclerosis patients. These complexes are characterized by smaller sizes and more negative electrical charge than native LDL and have pronounced atherogenic properties, as demonstrated by the accumulation of cholesterol esters in cultured smooth muscle cells and monocyte-derived macrophages. ${ }^{83}$ In vitro studies have demonstrated that LDL(-) had a cytotoxic effect on the EC, stimulating apoptosis and cytokine production, including IL-8, MCP-1, and VCAM-1.49,84,85 Incubation of cultured human monocytes with $\operatorname{LDL}(-)$, but not with LDL(+), resulted in cytokine release mediated by TLR4 and CD14, which was the main receptor recognizing $\operatorname{LDL}(-) .{ }^{86}$ Similar cytokine-inducing effect has also been described

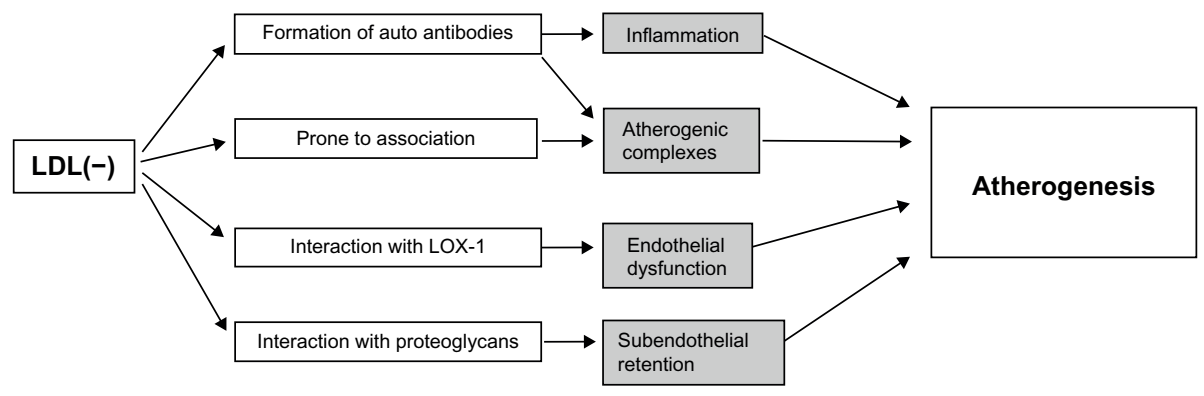

Figure 2 Atherogenic properties of electronegative LDL.

Notes: Several features account for the increased atherogenicity of electronegative LDL [LDL(-)]: formation of autoantibodies and susceptibility to self-association lead to formation of complexes with high atherogenicity. LDL(-) can cause endothelial dysfunction through interaction with LOX-I receptors. Interaction of LDL(-) with proteoglycans in the subendothelial intima causes increased retention of the particles that serve as a source of lipid storage. Abbreviations: LDL(-), electronegative low-density lipoprotein; LOX-I, LDL receptor I. 
for the ceramide-enriched LDL, which shares most of the properties of $\operatorname{LDL}(-) .{ }^{87,88}$

\section{Clinical importance of the LDL electronegativity index in prediction of CVD}

Recent studies strongly suggest that LDL electronegativity can be considered as an important factor in evaluating cardiovascular risk in many pathological conditions. As mentioned earlier, the elevated levels of electronegative LDL fractions have been reported in many conditions associated with high cardiovascular risk, including hyperlipidemia, diabetes, renal disease, and coronary syndromes. In patients with hypercholesterolemia, the most electronegative, mildly oxidized L5 LDL fraction was increased in comparison with healthy individuals. ${ }^{49}$ Elevated L5 LDL fraction has also been reported in familial hypercholesterolemia patients ${ }^{50}$ and in smokers, ${ }^{51}$ both cases being associated with high cardiovascular risk. Recent studies demonstrated that L5 LDL fraction was significantly increased in patients with ST-segment elevation myocardial infarction, where it was demonstrated to play a role in platelet activation and thrombosis. ${ }^{52,53}$ Elevated levels of L5 were observed in the plasma of patients with metabolic syndrome, a group of metabolic abnormalities that are associated with CVDs, which is used to identify individuals with high cardiovascular risk. ${ }^{89}$ Together these results suggest that LDL electronegativity might be considered as a novel predictor of cardiovascular risk.

The clinical importance of LDL electronegativity index has recently been explored in a study analyzing the correlation between the plasma level of L5 fraction and cardiovascular risk estimated using the Framingham risk score. ${ }^{90}$ This score has been developed during a large epidemiological Framingham Heart Study and is calculated using such predictors as sex, age, blood pressure, treatment of hypertension and diabetes mellitus, smoking, body mass index, and levels of total cholesterol and HDL. ${ }^{91,92}$ According to this classification, "general" CVD risk included coronary death, myocardial infarction, coronary insufficiency, angina, ischemic and hemorrhagic stroke, transient ischemic attack, peripheral artery disease, and heart failure. The "hard" CVD category included coronary death, myocardial infarction, and stroke. The study was performed on patients with metabolic syndrome and healthy individuals. Comparison of these two groups revealed no statistically significant difference in total cholesterol and LDL levels, whereas L5 fraction was significantly higher in patients with metabolic syndrome. No association was registered between L5 level and total cholesterol or LDL. Regression analysis demonstrated association between L5 content and fasting plasma glucose level and body mass index. L5 level and waist circumference were associated with CVD risks, and the independent contribution of L5 content (with controlled variance of waist circumference) was $11 \%$ of 30 -year "general" CVD risk and $8 \%$ of 30 -year "hard" CVD risk. Therefore, L5 content was shown to strongly correlate with different CVD risk factors and with CVD risk. Moreover, the authors argued that plasma L5 levels in asymptomatic individuals with metabolic syndrome also correlated with the number of fulfilled metabolic syndrome criteria and therefore with CVD progression. However, it has not been studied, whether or not the elevated L5 fraction was accompanied by an increase of other known atherogenic LDL modifications. Future studies should compare the diagnostic values of electronegative LDL and other atherogenic LDL types, including modified LDL fractions, such as oxidized LDL and desialylated LDL. Quantitative analysis of various types of modified LDL currently remains challenging because of low amounts of circulating particles. For instance, the association of atherogenic oxidized LDL with CVD risk was difficult to establish, making its use as a biomarker inconvenient. ${ }^{93}$ Therefore, LDL electronegativity was suggested as a novel index for CVD prediction because it is relatively easy to assess, although more studies are needed to strengthen this concept.

It remains to be determined whether the elevated level of L5 LDL actually plays a causative role in CVD. Nevertheless, properties of electronegative LDL attracted attention as potential direction of therapy improvement. As elevated levels of L5 are associated with such conditions as smoking, they may partly be ameliorated by lifestyle corrections that are generally recommended for individuals with high CVD risk. More directed therapeutic strategies emerge as a result of better understanding of electronegative LDL pathogenicity. In vitro studies on cultured human aortic epithelial cells explored the possibility of blocking L5 internalization through LOX-1 receptor using the naturally occurring compound sesamol. Study of the sesamol effect on Syrian hamsters fed a high-fat diet demonstrated that the compound addition could reduce plasma L5 levels and atherosclerotic lesion size. ${ }^{94}$ These results encourage the search for compounds specifically targeting the electronegative LDL fraction and related signaling. In another study, the authors demonstrated a decrease of LDL(-) uptake by macrophages and foam cell formation caused by anti-LDL(-) single-chain variable antibody fragments ( $\mathrm{scFv}$ ). The exposure of macrophages to LDL(-) resulted in enhanced 
expression of CD36, which promoted the lipid uptake and foam cell formation. ${ }^{95}$ The addition of the fragments led to a dose-dependent inhibition of the LDL(-) uptake as well as to the decrease of $C d 36$ expression at mRNA level. The protective effect of the fragments has also been demonstrated on animal model (Ldlr knockout mice), where it caused reduction of atherosclerotic lesion at the aortic sinus in comparison with untreated animals. ${ }^{96}$ However, it is important to evaluate the effects of agents specifically targeting electronegative LDL on the production, metabolism, and uptake of other atherogenic LDL subtypes.

The increase of LDL electronegativity can therefore be considered as an emerging independent risk factor of CVD, as confirmed by the analysis of various groups of patients with elevated CVD risk. The possibility of targeted therapy aiming to reduce the LDL(-) fraction has been explored in experiments on animal models. More studies are needed however to confirm the clinical utility of LDL electronegativity index on a larger scale and to design appropriate therapeutic approaches for reducing $\operatorname{LDL}(-)$ fraction in humans.

\section{Acknowledgment}

The work was supported by Russian Foundation for Basic Research (Grant number 15-04-09279).

\section{Disclosure}

The authors report no conflicts of interests in this work.

\section{References}

1. Krauss RM. Lipoprotein subfractions and cardiovascular disease risk. Curr Opin Lipidol. 2010;21:305-311.

2. Mills EJ, Rachlis B, Wu P, et al. Primary prevention of cardiovascular mortality and events with statin treatments: a network meta-analysis involving more than 65,000 patients. J Am Coll Cardiol. 2008;52: 1769-1781.

3. Goff DC Jr, Lloyd-Jones DM, Bennett G, et al. 2013 ACC/AHA guideline on the assessment of cardiovascular risk: a report of the American College of Cardiology/American Heart Association Task Force on Practice Guidelines. J Am Coll Cardiol. 2014;63:2935-2959.

4. Baigent C, Keech A, Kearney PM, et al. Efficacy and safety of cholesterol-lowering treatment: prospective meta-analysis of data from 90,056 participants in 14 randomised trials of statins. Lancet. 2005;366:1267-1278.

5. Afilalo J, Majdan AA, Eisenberg MJ. Intensive statin therapy in acute coronary syndromes and stable coronary heart disease: a comparative meta-analysis of randomised controlled trials. Heart. 2007;93:914-921.

6. Kjekshus J, Apetrei E, Barrios V, et al. Rosuvastatin in older patients with systolic heart failure. $N$ Engl J Med. 2007;357:2248-2261.

7. Gissi HFI, Tavazzi L, Maggioni AP, et al. Effect of rosuvastatin in patients with chronic heart failure (the GISSI-HF trial): a randomised, double-blind, placebo-controlled trial. Lancet. 2008;372:1231-1239.

8. Berneis KK, Krauss RM. Metabolic origins and clinical significance of LDL heterogeneity. J Lipid Res. 2002;43:1363-1379.

9. Mello AP, da Silva IT, Abdalla DS, et al. Electronegative low-density lipoprotein: origin and impact on health and disease. Atherosclerosis. 2011;215:257-265.
10. Rizzo M, Berneis K. Low-density lipoprotein size and cardiovascular risk assessment. QJM. 2006;99:1-14.

11. Packard C, Caslake M, Shepherd J. The role of small, dense low density lipoprotein (LDL): a new look. Int J Cardiol. 2000;74(Suppl 1): S17-S22.

12. Packard CJ. Small dense low-density lipoprotein and its role as an independent predictor of cardiovascular disease. Curr Opin Lipidol. 2006; $17: 412-417$.

13. Griffin BA, Caslake MJ, Yip B, et al. Rapid isolation of low density lipoprotein (LDL) subfractions from plasma by density gradient ultracentrifugation. Atherosclerosis. 1990;83:59-67.

14. Griffin BA, Freeman DJ, Tait GW, et al. Role of plasma triglyceride in the regulation of plasma low density lipoprotein (LDL) subfractions: relative contribution of small, dense LDL to coronary heart disease risk. Atherosclerosis. 1994;106:241-253.

15. Yee MS, Pavitt DV, Tan T, et al. Lipoprotein separation in a novel iodixanol density gradient, for composition, density, and phenotype analysis. J Lipid Res. 2008;49:1364-1371.

16. Ensign W, Hill N, Heward CB. Disparate LDL phenotypic classification among 4 different methods assessing LDL particle characteristics. Clin Chem. 2006;52:1722-1727.

17. Otvos JD, Jeyarajah EJ, Bennett DW, et al. Development of a proton nuclear magnetic resonance spectroscopic method for determining plasma lipoprotein concentrations and subspecies distributions from a single, rapid measurement. Clin Chem. 1992;38: 1632-1638.

18. Hirano T, Ito Y, Saegusa H, et al. A novel and simple method for quantification of small, dense LDL. J Lipid Res. 2003;44:2193-2201.

19. Ito Y, Fujimura M, Ohta M, et al. Development of a homogeneous assay for measurement of small dense LDL cholesterol. Clin Chem. 2011;57:57-65.

20. Albers JJ, Kennedy H, Marcovina SM. Evaluation of a new homogenous method for detection of small dense LDL cholesterol: comparison with the LDL cholesterol profile obtained by density gradient ultracentrifugation. Clin Chim Acta. 2011;412:556-561.

21. O’Neal D, Harrip P, Dragicevic G, et al. A comparison of LDL size determination using gradient gel electrophoresis and light-scattering methods. J Lipid Res. 1998;39:2086-2090.

22. Witte DR, Taskinen MR, Perttunen-Nio H, et al. Study of agreement between LDL size as measured by nuclear magnetic resonance and gradient gel electrophoresis. J Lipid Res. 2004;45:1069-1076.

23. Diffenderfer MR, Schaefer EJ. The composition and metabolism of large and small LDL. Curr Opin Lipidol. 2014;25:221-226.

24. Hirayama S, Miida T. Small dense LDL: an emerging risk factor for cardiovascular disease. Clin Chim Acta. 2012;414:215-224.

25. Steinberg D, Parthasarathy S, Carew TE, et al. Beyond cholesterol. Modifications of low-density lipoprotein that increase its atherogenicity. N Engl J Med. 1989;320:915-924.

26. Jaakkola O, Solakivi T, Tertov VV, et al. Characteristics of low-density lipoprotein subfractions from patients with coronary artery disease. Coron Artery Dis. 1993;4:379-385.

27. Tertov VV, Bittolo-Bon G, Sobenin IA, et al. Naturally occurring modified low density lipoproteins are similar if not identical: more electronegative and desialylated lipoprotein subfractions. Exp Mol Pathol. 1995;62:166-172.

28. Steinberg D, Witztum JL. Oxidized low-density lipoprotein and atherosclerosis. Arterioscler Thromb Vasc Biol. 2010;30:2311-2316.

29. Miller YI, Choi SH, Wiesner P, et al. Oxidation-specific epitopes are danger-associated molecular patterns recognized by pattern recognition receptors of innate immunity. Circ Res. 2011;108:235-248.

30. Pentikainen MO, Oorni K, Ala-Korpela M, et al. Modified LDL - trigger of atherosclerosis and inflammation in the arterial intima. J Intern Med. 2000;247:359-370.

31. Palinski W, Horkko S, Miller E, et al. Cloning of monoclonal autoantibodies to epitopes of oxidized lipoproteins from apolipoprotein E-deficient mice. Demonstration of epitopes of oxidized low density lipoprotein in human plasma. J Clin Invest. 1996;98:800-814. 
32. Itabe H, Yamamoto H, Imanaka T, et al. Sensitive detection of oxidatively modified low density lipoprotein using a monoclonal antibody. J Lipid Res. 1996;37:45-53.

33. Tertov VV, Sobenin IA, Orekhov AN. Modified (desialylated) low-density lipoprotein measured in serum by lectin-sorbent assay. Clin Chem. 1995;41:1018-1021.

34. Orekhov AN, Tertov VV, Mukhin DN. Desialylated low density lipoprotein - naturally occurring modified lipoprotein with atherogenic potency. Atherosclerosis. 1991;86:153-161.

35. Tertov VV, Kaplun VV, Sobenin IA, et al. Human plasma trans-sialidase causes atherogenic modification of low density lipoprotein. Atherosclerosis. 2001;159:103-115.

36. Younis N, Charlton-Menys V, Sharma R, et al. Glycation of LDL in non-diabetic people: small dense LDL is preferentially glycated both in vivo and in vitro. Atherosclerosis. 2009;202:162-168.

37. Soran H, Durrington PN. Susceptibility of LDL and its subfractions to glycation. Curr Opin Lipidol. 2011;22:254-261.

38. Hoff HF, Gaubatz JW. Isolation, purification, and characterization of a lipoprotein containing Apo B from the human aorta. Atherosclerosis. 1982;42:273-297.

39. Avogaro P, Bon GB, Cazzolato G. Presence of a modified low density lipoprotein in humans. Arteriosclerosis. 1988;8:79-87.

40. Sanchez-Quesada JL, Perez A, Caixas A, et al. Effect of glycemic optimization on electronegative low-density lipoprotein in diabetes relation to nonenzymatic glycosylation and oxidative modification. J Clin Endocrinol Metabol. 2001;86:3243-3249.

41. Griffin BA. Lipoprotein atherogenicity: an overview of current mechanisms. Proc Nutr Soc. 1999;58:163-169.

42. Matsui H, Okumura K, Toki Y, et al. Low-density lipoprotein particle size as an independent predictor of glycated low-density lipoprotein level. Diabetes Care. 1999;22:1220-1221.

43. La Belle M, Krauss RM. Differences in carbohydrate content of low density lipoproteins associated with low density lipoprotein subclass patterns. J Lipid Res. 1990;31:1577-1588.

44. Tribble DL, Rizzo M, Chait A, et al. Enhanced oxidative susceptibility and reduced antioxidant content of metabolic precursors of small, dense low-density lipoproteins. Am J Med. 2001;110:103-110.

45. Hasegawa G, Kajiyama S, Tanaka T, et al. The alpha-glucosidase inhibitor acarbose reduces the net electronegative charge of low-density lipoprotein in patients with newly diagnosed type 2 diabetes. Clin Chim Acta. 2008;390:110-114.

46. Tertov VV, Sobenin IA, Orekhov AN. Similarity between naturally occurring modified desialylated, electronegative and aortic low density lipoprotein. Free Radic Res. 1996;25:313-319.

47. Sanchez-Quesada JL, Benitez S, Otal C, et al. Density distribution of electronegative LDL in normolipemic and hyperlipemic subjects. J Lipid Res. 2002;43:699-705.

48. Greco G, Balogh G, Brunelli R, et al. Generation in human plasma of misfolded, aggregation-prone electronegative low density lipoprotein. Biophys J. 2009;97:628-635.

49. Chen $\mathrm{CH}$, Jiang T, Yang JH, et al. Low-density lipoprotein in hypercholesterolemic human plasma induces vascular endothelial cell apoptosis by inhibiting fibroblast growth factor 2 transcription. Circulation. 2003;107:2102-2108.

50. Yang CY, Raya JL, Chen HH, et al. Isolation, characterization, and functional assessment of oxidatively modified subfractions of circulating low-density lipoproteins. Arterioscler Thromb Vasc Biol. 2003;23:1083-1090.

51. Tang D, Lu J, Walterscheid JP, et al. Electronegative LDL circulating in smokers impairs endothelial progenitor cell differentiation by inhibiting Akt phosphorylation via LOX-1. J Lipid Res. 2008;49:33-47.

52. Chan HC, Ke LY, Chu CS, et al. Highly electronegative LDL from patients with ST-elevation myocardial infarction triggers platelet activation and aggregation. Blood. 2013;122:3632-3641.

53. Chang PY, Chen YJ, Chang FH, et al. Aspirin protects human coronary artery endothelial cells against atherogenic electronegative LDL via an epigenetic mechanism: a novel cytoprotective role of aspirin in acute myocardial infarction. Cardiovasc Res. 2013;99:137-145.
54. Schmitz G, Mollers C, Richter V. Analytical capillary isotachophoresis of human serum lipoproteins. Electrophoresis. 1997;18:1807-1813.

55. Zhang B, Kaneshi T, Ohta T, et al. Relation between insulin resistance and fast-migrating LDL subfraction as characterized by capillary isotachophoresis. J Lipid Res. 2005;46:2265-2277.

56. Damasceno NR, Sevanian A, Apolinario E, et al. Detection of electronegative low density lipoprotein (LDL-) in plasma and atherosclerotic lesions by monoclonal antibody-based immunoassays. Clin Biochem. 2006;39:28-38.

57. Santo Faulin Tdo E, de Sena KC, Rodrigues Telles AE, et al. Validation of a novel ELISA for measurement of electronegative low-density lipoprotein. Clin Chem Lab Med. 2008;46:1769-1775.

58. Faulin Tdo E, de Sena-Evangelista KC, Pacheco DB, et al. Development of immunoassays for anti-electronegative LDL autoantibodies and immune complexes. Clin Chim Acta. 2012;413:291-297.

59. Parasassi T, De Spirito M, Mei G, et al. Low density lipoprotein misfolding and amyloidogenesis. FASEB J. 2008;22:2350-2356.

60. Oorni K, Pentikainen MO, Ala-Korpela M, et al. Aggregation, fusion, and vesicle formation of modified low density lipoprotein particles: molecular mechanisms and effects on matrix interactions. J Lipid Res. 2000;41:1703-1714

61. Bancells C, Benitez S, Jauhiainen M, et al. High binding affinity of electronegative LDL to human aortic proteoglycans depends on its aggregation level. J Lipid Res. 2009;50:446-455.

62. Bancells C, Benitez S, Ordonez-Llanos J, et al. Immunochemical analysis of the electronegative LDL subfraction shows that abnormal $\mathrm{N}$-terminal apolipoprotein B conformation is involved in increased binding to proteoglycans. J Biol Chem. 2011;286:1125-1133.

63. Boren J, Olin K, Lee I, et al. Identification of the principal proteoglycanbinding site in LDL. A single-point mutation in apo-B100 severely affects proteoglycan interaction without affecting LDL receptor binding. J Clin Invest. 1998;101:2658-2664.

64. Sanchez-Quesada JL, Villegas S, Ordonez-Llanos J. Electronegative low-density lipoprotein. A link between apolipoprotein B misfolding, lipoprotein aggregation and proteoglycan binding. Curr Opin Lipidol. 2012;23:479-486.

65. Ke LY, Engler DA, Lu J, et al. Chemical composition-oriented receptor selectivity of L5, a naturally occurring atherogenic low-density lipoprotein. Pure Appl Chem. 2011;83(9).

66. Ursini F, Davies KJ, Maiorino M, et al. Atherosclerosis: another protein misfolding disease? Trends Mol Med. 2002;8:370-374.

67. Asatryan L, Hamilton RT, Isas JM, et al. LDL phospholipid hydrolysis produces modified electronegative particles with an unfolded apoB-100 protein. J Lipid Res. 2005;46:115-122.

68. Parasassi T, Bittolo-Bon G, Brunelli R, et al. Loss of apoB-100 secondary structure and conformation in hydroperoxide rich, electronegative LDL(-). Free Radic Biol Med. 2001;31:82-89.

69. Blanco FJ, Villegas S, Benitez S, et al. 2D-NMR reveals different populations of exposed lysine residues in the apoB-100 protein of electronegative and electropositive fractions of LDL particles. J Lipid Res. 2010;51:1560-1565.

70. Brunelli R, Balogh G, Costa G, et al. Estradiol binding prevents ApoB-100 misfolding in electronegative LDL(-). Biochemistry. 2010;49:7297-7302.

71. Benitez S, Sanchez-Quesada JL, Lucero L, et al. Changes in low-density lipoprotein electronegativity and oxidizability after aerobic exercise are related to the increase in associated non-esterified fatty acids. Atherosclerosis. 2002; 160:223-232.

72. Gaubatz JW, Gillard BK, Massey JB, et al. Dynamics of dense electronegative low density lipoproteins and their preferential association with lipoprotein phospholipase A(2). J Lipid Res. 2007;48:348-357.

73. Jayaraman S, Gantz DL, Gursky O. Effects of phospholipase A(2) and its products on structural stability of human LDL: relevance to formation of LDL-derived lipid droplets. J Lipid Res. 2011;52:549-557.

74. Benitez S, Sanchez-Quesada JL, Ribas V, et al. Platelet-activating factor acetylhydrolase is mainly associated with electronegative low-density lipoprotein subfraction. Circulation. 2003;108:92-96. 
75. Holopainen JM, Medina OP, Metso AJ, et al. Sphingomyelinase activity associated with human plasma low density lipoprotein. J Biol Chem. 2000;275:16484-16489.

76. Kinnunen PK, Holopainen JM. Sphingomyelinase activity of LDL: a link between atherosclerosis, ceramide, and apoptosis? Trends Cardiovasc Med. 2002;12:37-42.

77. Bancells C, Canals F, Benitez S, et al. Proteomic analysis of electronegative low-density lipoprotein. J Lipid Res. 2010;51:3508-3515.

78. Wyatt AR, Wilson MR. Identification of human plasma proteins as major clients for the extracellular chaperone clusterin. J Biol Chem. 2010;285:3532-3539.

79. Benitez S, Villegas V, Bancells C, et al. Impaired binding affinity of electronegative low-density lipoprotein (LDL) to the LDL receptor is related to nonesterified fatty acids and lysophosphatidylcholine content. Biochemistry. 2004;43:15863-15872.

80. Urata J, Ikeda S, Koga S, et al. Negatively charged low-density lipoprotein is associated with atherogenic risk in hypertensive patients. Heart Vessels. 2012;27:235-242.

81. Lu J, Yang JH, Burns AR, et al. Mediation of electronegative low-density lipoprotein signaling by LOX-1: a possible mechanism of endothelial apoptosis. Circ Res. 2009;104:619-627.

82. Chu CS, Wang YC, Lu LS, et al. Electronegative low-density lipoprotein increases C-reactive protein expression in vascular endothelial cells through the LOX-1 receptor. PloS One. 2013;8:e70533.

83. Sobenin IA, Salonen JT, Zhelankin AV, et al. Low density lipoproteincontaining circulating immune complexes: role in atherosclerosis and diagnostic value. BioMed Res Int. 2014;2014:205697.

84. Sanchez-Quesada JL, Camacho M, Anton R, et al. Electronegative LDL of FH subjects: chemical characterization and induction of chemokine release from human endothelial cells. Atherosclerosis. 2003;166:261-270

85. Benitez S, Camacho M, Bancells C, et al. Wide proinflammatory effect of electronegative low-density lipoprotein on human endothelial cells assayed by a protein array. Biochim Biophys Acta. 2006;1761:1014-1021.
86. Estruch M, Bancells C, Beloki L, et al. CD14 and TLR4 mediate cytokine release promoted by electronegative LDL in monocytes. Atherosclerosis. 2013;229:356-362.

87. Estruch M, Sanchez-Quesada JL, Ordonez-Llanos J, et al. Ceramide-enriched LDL induces cytokine release through TLR4 and CD14 in monocytes. Similarities with electronegative LDL. Clin Invest Arterioscler. 2014;26:131-137.

88. Estruch M, Sanchez-Quesada JL, Beloki L, et al. The induction of cytokine release in monocytes by electronegative low-density lipoprotein (LDL) is related to its higher ceramide content than native LDL. Int J Mol Sci. 2013;14:2601-2616.

89. Lee AS, Chen WY, Chan HC, et al. Gender disparity in LDL-induced cardiovascular damage and the protective role of estrogens against electronegative LDL. Cardiovasc Diabetol. 2014;13:64.

90. Hsu JF, Chou TC, Lu J, et al. Low-density lipoprotein electronegativity is a novel cardiometabolic risk factor. PLoS One. 2014;9:e107340.

91. Pencina MJ, D'Agostino RB Sr, Larson MG, et al. Predicting the 30-year risk of cardiovascular disease: the framingham heart study. Circulation. 2009;119:3078-3084.

92. D’Agostino RB Sr, Vasan RS, Pencina MJ, et al. General cardiovascular risk profile for use in primary care: the Framingham Heart Study. Circulation. 2008;117:743-753.

93. Fraley AE, Tsimikas S. Clinical applications of circulating oxidized low-density lipoprotein biomarkers in cardiovascular disease. Curr Opin Lipidol. 2006;17:502-509.

94. Chen WY, Chen FY, Lee AS, et al. Sesamol reduces the atherogenicity of electronegative L5 LDL in vivo and in vitro. J Nat Prod. 2015;78: 225-233.

95. Pedrosa AM, Faine LA, Grosso DM, et al. Electronegative LDL induction of apoptosis in macrophages: involvement of Nrf2. Biochim Biophys Acta. 2010;1801:430-437.

96. Kazuma SM, Cavalcante MF, Telles AE, et al. Cloning and expression of an anti-LDL(-) single-chain variable fragment, and its inhibitory effect on experimental atherosclerosis. MAbs. 2013;5:763-775.
Vascular Health and Risk Management

\section{Publish your work in this journal}

Vascular Health and Risk Management is an international, peerreviewed journal of therapeutics and risk management, focusing on concise rapid reporting of clinical studies on the processes involved in the maintenance of vascular health; the monitoring, prevention and treatment of vascular disease and its sequelae; and the involvement of

\section{Dovepress}

metabolic disorders, particularly diabetes. This journal is indexed on PubMed Central and MedLine. The manuscript management system is completely online and includes a very quick and fair peer-review system, which is all easy to use. Visit http://www.dovepress.com/ testimonials.php to read real quotes from published authors. 